and then send their measurements to a central database. Students can also study light sources in their neighbourhood, using simple transmission diffraction gratings-a good cloudy-night activity!

Light pollution also hinders the recruitment, training, and work of amateur (volunteer) astronomers. Amateurs make important contributions to astronomy research and education. Amateurs in many parts of the world -including Japan-have carried out important studies of light pollution, thanks to their skill and motivation. They are our allies - "grass-roots" voices for the preservation of the astronomical environment.

The issue of space debris is somewhat more difficult, since we have no control in the matter; most people, however, can relate to the problem, because of widespread media reporting of space activities. The issue of the pollution of the electromagnetic spectrum is even more difficult, because people have no direct experience with it, and they somehow feel that scientists can overcome any technological problem of this kind. Public education is an essential part of any solution.

What can we do about light pollution? We cannot just replace the real sky by the planetarium sky, or the computer screen-useful as these may be. We cannot expect people to travel to dark sites, even though the rich and motivated can do so. We cannot do all astronomy from space, even though this is a popular misconception. We must promote astronomy education and culture, and encourage our colleagues and students to do the same. We must educate ourselves about the preservation of the astronomical environment, and "spread the word". We must work with partners: teachers, amateur astronomers, lighting engineers, and the media, to achieve these goals. We must support organizations such as IAU Commission 50, and the International Dark-Sky Association. We must promote an appreciation of the night sky by making skygazing a part of our courses, and other activities. Few people who have seen a dark sky are unmoved.

\title{
4. NATURAL OPTICAL SKY BACKGROUND
}

Ch. Leinert ${ }^{a}$ and K. Mattila ${ }^{b}\left({ }^{a}\right.$ Max-Planck-Institut für Astronomie, Heidelberg, Germany;

${ }^{b}$ University of Helsinki Observatory, Finland)

\subsection{INTRODUCTION}

Knowledge of the natural dark night sky brightness is required if one wants to set meaningful limits to the disturbance by artificial illumination. Knowledge of the behaviour of the natural dark night sky brightness is also required if one wants to monitor the sky for existing or changing light pollution. Therefore, before coming to quantitative results of night sky brightness measurements, we give an overview on the different components of night sky brightness and their different behaviour. From this, recommendations will result for the planning of future sky brightness monitoring programmes, as they are requested in the first of the proposed IAU resolutions to result from this joint discussion.

\subsection{BRIGHTNESS OF THE COMPONENTS OF THE NIGHT SKY}

The light of the night sky comes from several "layers" at vastly different distances. Neglecting the small contribution from extragalactic background light and the diffuse galactic light, which approximately can be taken as a $20 \%$ addition to the light of the stars, the main contributors to the night sky brightness are--the integrated starlight due to our own galaxy $\left(15-200 \mathrm{~S}_{10}\left[1 \mathrm{~S}_{10}\right.\right.$ unit $=$ one $10^{\text {th }}$ magnitude star per square degree $=2 \cdot 17 \cdot 10^{-8} \mathrm{~W} / \mathrm{m}^{2} \mathrm{sr} \mu \mathrm{m}$ at B.]), -the zodiacal light, due to scattering of solar radiation on interplanetary dust $\left(30-100 \mathrm{~S}_{10}\right)$, airglow emission from the high atmospheric layers $\left(50-180 \mathrm{~S}_{10}\right)$, and scattering of these components in the troposphere. The light pollution, of course, results from troposheric backscattering. Tropospheric scattering contains two components: Rayleigh scattering, with almost isotropic distribution of scattered brightness, and Mie scattering by aerosols, preferentially scattering into the forward direction $\pm 30^{\circ}$. These two components are present in the artificial sky illumination as well, i.e. the observable sky brightening towirds a city is not the whole story. There is an almost isotropic Rayleigh component as well!

\subsection{TEMPORAL VARIATIONS}

Most of the variations are due to Airglow, sometimes by a gradual decrease during the night, but fluctuations and continuous brightness increases occur as well. Differences fron night to night. 
may be substantial (20-50\%), and this is a most significant effect to be considered when measuring changes in light pollution!. The variations with solar cycle are of similar size $(\approx 50 \%)$ and correlated to the solar $10.7 \mathrm{~cm}$ flux.

There are indirect temporal variations caused by the passage of Milky Way and the zodiacal light, which may introduce spurious variations in measurements taken at different hours or days. Some change in scattered light intensity will also occur by the diurnal motion of these extended light sources.

There is a slight annual variation of zodiacal light, by about $\pm 10 \%$ at high ecliptic latitudes, due to the Earth's orbital motion within the interplanetary dust cloud.

\subsection{BRIGHTNESS OF THE NIGHT SKY}

Measurements of dark night sky brightness at different observatories lead to best values of typically $22 \mathrm{mag} / \mathrm{square} \operatorname{arcsec}$ in $\mathrm{V}, 23 \mathrm{mag} / \mathrm{square} \operatorname{arcsec}\left(\approx 100 \mathrm{~S}_{10}\right)$ in $\mathrm{B}$. The spectrum of the night sky is dominated by strong airglow lines e.g. at $557.7 \mathrm{~nm}[\mathrm{OI}], 589 \mathrm{~nm}[\mathrm{NaI}], 630 \mathrm{~nm}[\mathrm{OI}]$ and $\mathrm{OH}$ emission at longer wavelengths. Comparatively dark spectral bands are from $380 \mathrm{~nm}$ to $400 \mathrm{~nm}$, $450 \mathrm{~nm}$ to $520 \mathrm{~nm}, 640 \mathrm{~nm}$ to $710 \mathrm{~nm}$, and around $820 \mathrm{~nm}$. If a choice has to be made, try to protect these bands!

\subsection{RECOMMENDATIONS FOR LIGHT POLLUTION MEASUREMENTS}

Therefore, if one wants to avoid mixing up natural changes with man-made increases in monitoring of light pollution, the following measures are recommended:

- Perform measurements at preselected, known positions on the sky, document all relevant coordinates, avoid stars or correct for them.

- Measurements should cover a sufficiently long interval (several hours per night/ several nights) to be free of "random" airglow variations within a night and from night to night.

- Measurements should cover a substantial fraction of the solar 11-year cycle or, at least, take the effect of solar activity into account when comparing measurements from different years.

\section{FIELD SURVEY OF OUTDOOR LIGHTING IN JAPAN}

\section{K. Narisada, K. Kawakami}

The Illuminating Engineering Institute of Japan conducted a field survey concerning the outdoor lighting in 1995, in various areas of the country. The aim of the survey was to find which types of luminaires in existing lighting installations are wasting the greatest amount of upward flux into the sky. The survey was conducted in six of the seven zones, proposed in an earlier draft of the guide of the CIE, i.e. E1, E2a, E3a, E3b, E4a and E4b. On the basis of the results of the survey, the proportion of the upward flux per 100 hectares was compared separately for eight types of luminaires, such as the luminaires for traffic roads, residential areas, decorative (shopping) streets, projectors, luminous signs, etc. As a result, it was found that the major sources of the upward flux were the luminaires for decorative streets and luminous signs and to be improved their photometric characteristics.

\section{BILATERAL AGREEMENTS ON LIMITS TO OUTDOOR LIGHTING; THE NEW CIE RECOMMENDATIONS, THEIR ORIGIN AND IMPLICATIONS}

\section{A. Schreuder}

\section{Summary}

Sky glow presents itself as a background luminance over the sky, against which the astronomical objects are to be observed. The interference of astronomical observations is caused by the resulting reduction in luminance contrast. The glow is caused by non-directional scatter of light by particles in space and in the atmosphere. Part of the light, and part of the particles are natural, and part is man-made. The '(natural) background radiation' is defined as the radiation (luminance) resulting from the scatter of natural light by natural particles. For earth-bound observatories, the background luminance is the limit for sky glow. It is customary to express the sky glow (from man-made sources) as a percentage of the natural background luminance. 